\title{
Numerical simulation of gas-phonon coupling in thermal transpiration flows
}

\author{
Xiaohui Guo, ${ }^{1}$ Dhruv Singh, ${ }^{2}$ Jayathi Murthy, ${ }^{2}$ and Alina A. Alexeenko ${ }^{1}$ \\ ${ }^{1}$ School of Aeronautics and Astronautics, Purdue University, West Lafayette, Indiana 47907, USA \\ ${ }^{2}$ School of Mechanical Engineering, Purdue University, West Lafayette, Indiana 47907, USA
}

(Received 10 March 2009; published 15 October 2009)

\begin{abstract}
Thermal transpiration is a rarefied gas flow driven by a wall temperature gradient and is a promising mechanism for gas pumping without moving parts, known as the Knudsen pump. Obtaining temperature measurements along capillary walls in a Knudsen pump is difficult due to extremely small length scales. Meanwhile, simplified analytical models are not applicable under the practical operating conditions of a thermal transpiration device, where the gas flow is in the transitional rarefied regime. Here, we present a coupled gas-phonon heat transfer and flow model to study a closed thermal transpiration system. Discretized Boltzmann equations are solved for molecular transport in the gas phase and phonon transport in the solid. The wall temperature distribution is the direct result of the interfacial coupling based on mass conservation and energy balance at gas-solid interfaces and is not specified a priori unlike in the previous modeling efforts. Capillary length scales of the order of phonon mean free path result in a smaller temperature gradient along the transpiration channel as compared to that predicted by the continuum solid-phase heat transfer. The effects of governing parameters such as thermal gradients, capillary geometry, gas and phonon Knudsen numbers and, gas-surface interaction parameters on the efficiency of thermal transpiration are investigated in light of the coupled model.
\end{abstract}

DOI: 10.1103/PhysRevE.80.046310

PACS number(s): 47.61.Cb, 47.45.Gx, 51.20.+d, 51.90.+r

\section{INTRODUCTION}

Growing interest in micro/nanoscale science and technologies have led to the development of many microscale devices such as microsensors [1], microactuators [2], microbiological devices [3], microscale chemical analyzers [4], and micropropulsion systems [5]. In many of these applications, a reliable micro/nanosize gas pump is required. Compared to conventional pumping methods, thermal transpiration has been considered as one attractive alternative gas pumping mechanism for microdevices [6-8] as it does not employ any moving parts.

Thermal transpiration, also known as thermal creep, was originally observed in experiments by Reynolds in the late nineteenth century [9]. In 1910, Knudsen [10] continued the experimental investigations and gave a qualitative explanation of thermal creep flow as shown in Fig. 1. Later, West published a mathematical derivation of the origin of thermal transpiration [11].

Until recently thermal transpiration flows have been studied principally in high-vacuum applications [12-14]. In the 1990 s, studies of thermal transpiration phenomena have been reinvigorated in the scene of growing interest in MEMS technology. Numerous relevant work followed up concurrently in the experimental, analytical and numerical analysis aspects [6,15-18]. However, most theoretical and numerical models of thermal transpiration are limited to the consideration of gas phase only. Such models assume a wall temperature distribution specified a priori, e.g., linear, which is being used as a boundary condition. The prime problem lying here is that the conjugate heat transfer at gas-solid interfaces is ignored. The coupling between gas and solid phases can play a big role when gas flows are highly rarefied in transitional regime. For instance, a detailed discussion on subcontinuum thermal transport across semiconductor-gas interfaces can be found in reference [19].
In this work, a coupled gas-phonon model has been used to study numerically the thermal transpiration in a closed single-stage Knudsen compression system. The finite-volume discretization of the Boltzmann transport equation has been applied to both molecules in the gas phase and phonons in the solid phase. In particular, molecular velocity distribution functions using the ES (ellipsoidal statistical) BhatnagarGross-Krook (BGK) model [20] and phonon mean energy densities using the gray-BTE (Boltzmann Transport Equation) model [21] will be calculated simultaneously. The interfacial temperature is found by considering mass conservation and energy balance at contact surfaces. With this coupled model, it has been found (and quantified) for the first time that the temperature gradient for thermal transpiration can be much less than that based on the nominal difference between reservoirs. In fact, there are significant temperature jumps at both sides of contact surfaces in the presence of non-negligible rarefaction effects of gas and ballistic effects of phonons. It is known that the total pressure rise and the mass flow rate in a Knudsen pump are closely related to the wall temperature gradients. The former is of particular interest for closed systems while the latter is important for open systems.

In following sections, governing equations and the gasphonon interaction model is introduced first followed by the

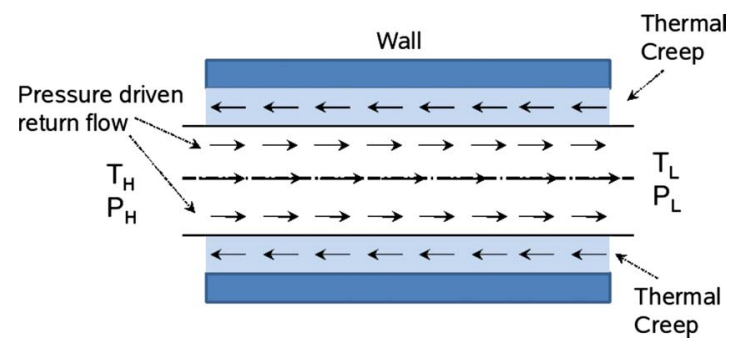

FIG. 1. (Color online) Thermal transpiration flow. 
description of the numerical method. Model verification is done by comparison between numerical solutions and experimental or analytical results for one-dimensional (1D) Fourier flow, Couette flow, and a one-dimensional gas-phonon heat transfer problem. Next, we explain simulation setup, analyze the asymptotic solutions and summarize the material properties and system conditions. In the results and discussion section, we show the importance of interfacial coupling effects for different models. A discussion on temperature gradient, device geometries, Knudsen numbers and surface conditions is also presented. Finally, we compare the results from two theoretical gas-phase models with that from the coupled simulation for a closed thermal transpiration system.

\section{MODELS AND NUMERICAL METHODS}

\section{A. Governing equations for gas phase}

The steady state Boltzmann kinetic equation is used to describe movements and collisions of gas molecules in the phase space. By substituting the collision integral with a simplified BGK-type operator, the governing equation in terms of gas velocity distribution function $f(\vec{x}, \vec{v})$ can be written as [20],

$$
\vec{v} \cdot \nabla f=\frac{f_{0}-f}{\tau_{g}},
$$

where $\vec{x}$ stands for the physical space, $\vec{v}$ stands for the molecular velocity space, and $1 / \tau_{g}$ refers to the collision frequency. In the standard BGK model, $f_{0}$ is the local equilibrium Maxwellian distribution [22],

$$
f_{0}=\frac{n}{\sqrt{(2 \pi R T)^{3}}} \exp \left(-\frac{\vec{v}^{\prime 2}}{2 R T}\right),
$$

where $\overrightarrow{v^{\prime}}=\vec{v}-\vec{v}_{0}$ is the thermal velocity, and $\vec{v}_{0}$ is the average flow velocity. In the ES-BGK model, $f_{0}$ is the local anisotropic Gaussian distribution,

$$
\begin{gathered}
f_{0}=\frac{n}{\sqrt{(2 \pi)^{3} \operatorname{det}\left[\lambda_{i j}\right]}} \exp \left[-\left(\lambda_{i j}\right)^{-1} v_{i} v_{j}\right] \\
\lambda_{i j}=\frac{1}{\operatorname{Pr}} R T \delta_{i j}+\left(1-\frac{1}{\operatorname{Pr}}\right) \frac{p_{i j}}{\rho},
\end{gathered}
$$

where $p_{i j}$ is the pressure tensor, $\lambda_{i j}$ is the anisotropic multiplier and Pr is the Prandtl number. For example, Pr is $2 / 3$ for a monatomic gas. A BGK model kinetic equation is not suitable for simulations of thermal transpiration as they inhibit the equilibrium characteristic to be isotropic and fail to correctly reproduce the diffusion coefficient for momentum and heat equation simultaneously on the continuum end [23]. Furthermore, since the working temperature of the gas is nominally around room temperature, internal energy and rotational energy of gas molecules is always in equilibrium with their average translational energy and transport may be well described under the ES-BGK approximation of the collision integral. For two-dimensional problems, reduced distribution functions $f_{1}$ and $f_{2}$ [24] are used to offset the com- putational cost. In the presented model, molecular viscosity is simulated using a power-law dependence on temperature [25],

$$
\mu(T)=\mu_{r e f}\left(\frac{T}{T_{r e f}}\right)^{\beta},
$$

where $\mu_{\text {ref }}$ is the reference viscosity at reference temperature $T_{r e f}$ and $\beta$ is the viscosity-temperature exponent. According to the gas kinetic theory, the thermal conductivity $k_{g}$ for gas is,

$$
k_{g}(T)=\frac{5}{2} \frac{R \mu(T)}{\operatorname{Pr}}
$$

which is also a temperature dependent variable.

It is worth paying special attention to the fact that the velocity space is always three-dimensional even for twodimensional and one-dimensional problems. For example, the phase space will still have four independent variables in total even for a one-dimensional problem. However, it is possible to relieve the computational complexity by using reduced distribution functions (rdf) by considering the symmetry in geometry. Take for example a two-dimensional problem with $w$ being the molecular velocity in the $z$ direction. As the bulk velocity must be zero in the third direction due to symmetry, i.e., $w_{0}=0$, the original distribution function $f$ can be replaced by its reduced forms $f_{1}$ and $f_{2}$ as following,

$$
\begin{gathered}
f_{1}=\int_{-\infty}^{\infty} f \cdot d w \\
f_{2}=\int_{-\infty}^{\infty} f \cdot w^{2} d w .
\end{gathered}
$$

The following simplification can be made when the distribution function $f$ is a Maxwellian:

$$
\begin{gathered}
f_{10}=\frac{n}{2 \pi R T} \exp \left(-\frac{\vec{v}^{\prime 2}}{2 R T}\right) \\
f_{20}=\frac{n}{2 \pi} \exp \left(-\frac{\vec{v}^{\prime 2}}{2 R T}\right) .
\end{gathered}
$$

The relative magnitude of the collision relaxation term is characterized by the Knudsen number, the definition of which based on the hard-sphere model [26] can be written as,

$$
\mathrm{Kn}_{g}=\frac{\lambda_{g}}{L_{r e f}}=\frac{\mu}{p L_{r e f}} \sqrt{\frac{\pi R T}{2}},
$$

where $\lambda_{g}$ is the gas molecular mean-free path (MFP) and $L_{r e f}$ is the characteristic length. For channels and capillaries, it is conventional to use the hydraulic diameter $D_{h}$ as the reference length $L_{r e f}$. The definition of the hydraulic diameter is [25],

$$
L_{r e f}=D_{h}=\frac{4 A}{U},
$$

where $U$ is the wetted perimeter. For example, the hydraulic diameter for a three-dimensional channel with a rectan- 
gular cross-section is $L_{r e f}=2 H /(H / W+1)$, where $H / W$ is the aspect ratio. By substituting $H / W=0$, for two-dimensional cases one obtains a reference length, which is twice the channel height, i.e., $L_{r e f}=2 H$.

\section{B. Governing Equations for Solid-phase}

Based on the phonon energy density $e^{\prime \prime}$, a similar kinetic equation for solid can be written as [21],

$$
\begin{gathered}
v_{g} \vec{s} \cdot \nabla e^{\prime \prime}=\frac{e_{0}-e^{\prime \prime}}{\tau} \\
e^{\prime \prime}=\frac{1}{4 \pi} \sum_{q} \int f_{p} \hbar \omega D(\omega) d \omega ; \quad e_{0}=\frac{1}{4 \pi} \int_{4 \pi} e^{\prime \prime} d \Omega,
\end{gathered}
$$

where $e^{\prime \prime}(\vec{x}, \vec{s})$ is the energy density of phonons (integrated over all frequencies $\omega$, summed over all phonon polarizations $q$ ) at position $\vec{x}$ in wave-vector direction $\vec{s}$. Here, $e_{0}(\vec{x})$ represents the angular average of $e^{\prime \prime}(\vec{x}, \vec{s})$ over the solid angle $\Omega$. The gray-BTE model treats phonons of all polarizations $q$ and wave vectors as having the same group velocity $v_{g}$ with a single relaxation time $\tau$. The particular group velocity is usually chosen to be the dominant velocity of the major phonon group at the temperature of interest. Once $v_{g}$ is chosen, $\tau$ can be calculated from the bulk thermal conductivity known a priori,

$$
k_{p}=\frac{1}{3} C_{p} v_{g}^{2} \tau,
$$

where $C_{p}$ is the heat capacity for phonons.

A gray model of the phonon Boltzmann equation sufficiently captures the size effects, ballistic transport and thermal transport in the cross-plane direction [27]. We choose to use this approximation over more detailed models, such as those that incorporate frequency dependence in the light that no information on frequency dependence of gas-phonon interaction is available. Size effects in thermal transport within the solid is usually characterized by the term acoustic thickness [21] which is the inverse Knudsen number. One can write the phonon-phase Knudsen number as,

$$
\mathrm{Kn}_{p}=\frac{v_{g} \tau}{L_{r e f}}
$$

with $1 / \mathrm{Kn}_{p}$ being the phonon acoustic thickness. Both $\mathrm{Kn}_{g}$ and $\mathrm{Kn}_{p}$ characterize the coupled gas-phonon thermal transport flows.

\section{Macroscopic Parameters}

For the gas phase, we focus on the molecular number density $n$ (or mass density $\rho$ ), bulk velocities $\vec{v}_{0}$, temperature $T$, pressure $p$ (or the pressure tensor $p_{i j}$ ), and heat flux $\vec{Q}_{i}$. Relevant macroscopic observables for the solid are the temperature $T$ and heat flux $\vec{Q}_{i}$. The macroscopic parameters are given below with subscripts $g$ and $p$ denoting gas and phonons, respectively,

$$
n=\int_{-\infty}^{\infty} f d^{3} \vec{v}=\frac{\rho}{m}
$$

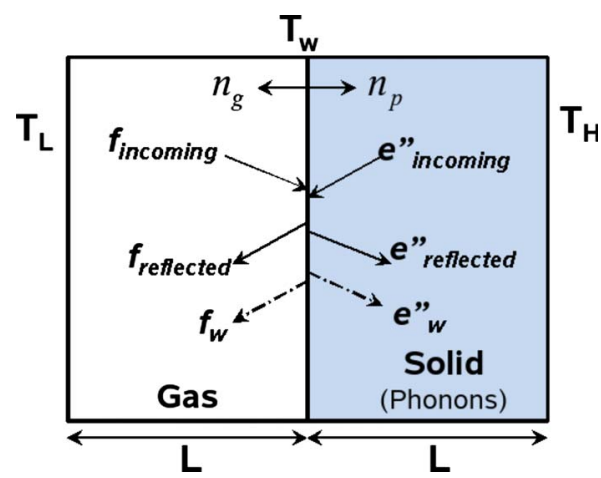

FIG. 2. (Color online) Schematic of gas-phonon interaction.

$$
\begin{gathered}
\vec{v}_{0}=\frac{1}{n} \int_{-\infty}^{\infty} f \vec{v} d^{3} \vec{v} \\
T_{g}=\frac{1}{3 R n} \int_{-\infty}^{\infty} f \vec{v}^{\prime 2} d^{3} \vec{v} \\
T_{p}=\frac{4 \pi}{C_{p}} e_{0}+T_{r e f} \\
p_{i j}=\frac{1}{n} \int_{-\infty}^{\infty} f v_{i}^{\prime} v_{j}^{\prime} d^{3} \vec{v} \\
\vec{Q}_{g}=\frac{m}{2 n} \int_{-\infty}^{\infty} f \vec{v}^{\prime} \vec{v}^{\prime 2} d^{3} \vec{v} \\
\vec{Q}_{p}=\int_{0}^{4 \pi} v T_{g} e^{\prime \prime} \vec{s} d \Omega .
\end{gathered}
$$

\section{Surface Interactions and Boundary Conditions}

At gas-phonon interfaces, conservation of mass, momentum and energy must be satisfied. In particular, the net energy flux from the gas phase should be balanced by that from the phonon side. Detailed explanation of the interfacial energy transfer, the non dimensional variables that arise out of it and its implementation are given in [19].

As seen in Fig. 2, the energy flux for each phase has two components, which are due to incident and reflected molecules and phonons. Therefore, the mathematical formulation of gas-phonon surface interaction is [19],

$$
\begin{gathered}
\left.\left(\vec{n} \cdot \overrightarrow{v_{0}}\right)\right|_{f_{w}, \vec{v} \cdot \overrightarrow{n_{g}}>0}=\left.\left(\vec{n} \cdot \overrightarrow{v_{0}}\right)\right|_{f, \vec{v} \cdot \overrightarrow{n_{g}}<0} \\
\alpha_{p} \cdot\left(\left.Q_{p}\right|_{e_{0 w}, \vec{s} \cdot \overrightarrow{n_{p}}>0}+\left.Q_{p}\right|_{e^{\prime \prime}, \vec{s} \cdot \overrightarrow{n_{p}}<0}\right) \\
=\sigma_{T, g} \cdot\left(\left.Q_{g}\right|_{f_{w}, \vec{v} \cdot \overrightarrow{n_{g}}>0}+\left.Q_{g}\right|_{f, \vec{v} \cdot \overrightarrow{n_{g}}<0}\right),
\end{gathered}
$$

where $\sigma_{g, T}$ is the gas-phase thermal accommodation coefficient based on the Maxwell specular-diffuse model and $\alpha_{p}$ is 

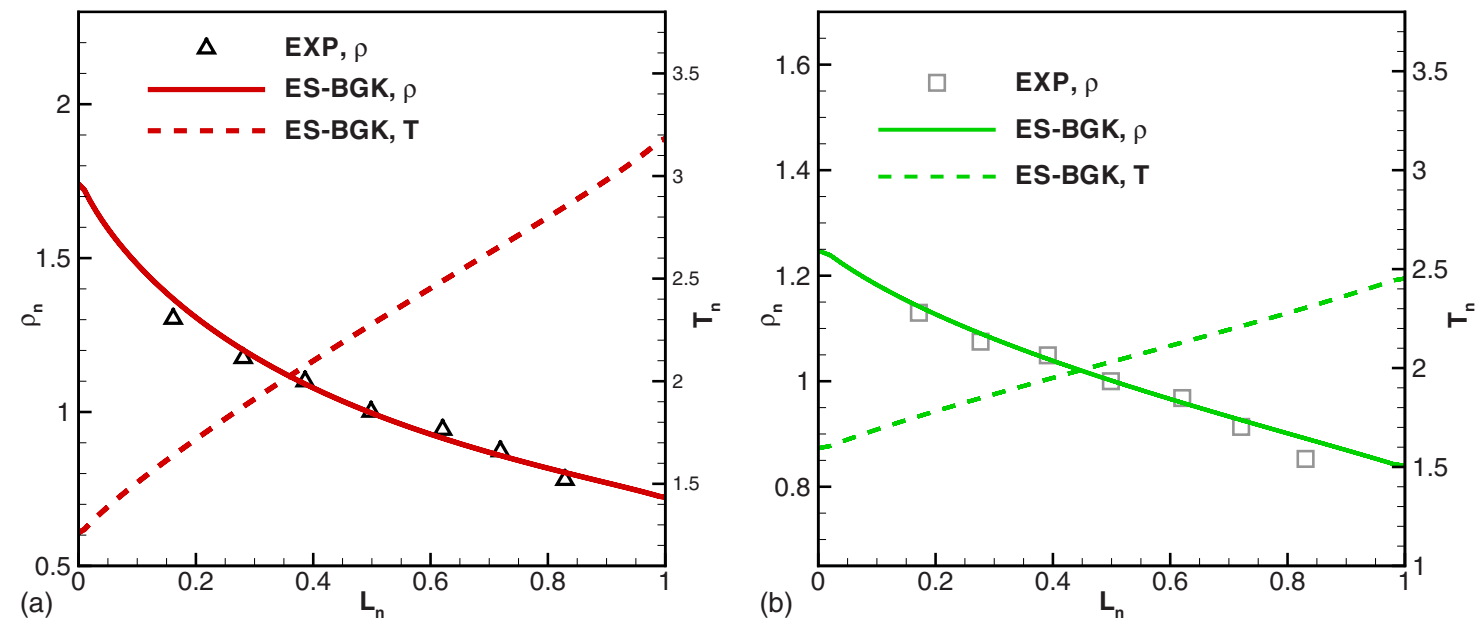

FIG. 3. (Color online) Comparisons of gas-phase 1D heat transfer with normalizations being consistent as in Ref. [30]for (a) Kn $=0.075$ and (b) $\mathrm{Kn}=0.399$.

the phonon transimissivity. In addition, $\vec{v} \cdot \overrightarrow{n_{p}}<0$ refers to incoming directions from solid to the wall, and $\vec{v} \cdot \overrightarrow{n_{p}}>0$ refers to outgoing directions from the wall to the solid domain; similar definitions are applied to $\vec{v} \cdot \overrightarrow{n_{g}}$.

The reduced distribution functions and energy density functions at the wall are given by,

$$
\begin{gathered}
f_{w, \vec{v} \cdot \overrightarrow{n_{g}}>0}=f_{\text {Maxwellian }}\left(T_{w}\right) \\
e_{0, \vec{s} \cdot \overrightarrow{n_{p}}>0}=\frac{C_{p}\left(T_{w}-T_{r e f}\right)}{4 \pi} \\
\nabla e_{\vec{s} \cdot \overrightarrow{n_{p}}<0}^{\prime \prime} \cdot \vec{s}=0 .
\end{gathered}
$$

The interfacial temperature distribution is updated based on Eqs. (25)-(29) until $\left|T_{w, k+1}-T_{w, k}\right|$ ( $k$ being the iteration index) falls below a specific tolerance $\epsilon$.

\section{E. Numerical Method}

In order to reduce computational round-off errors, all parameters have been normalized. For Eqs. (1) and (13), additional Knudsen numbers will appear on the left-hand side, as $\mathrm{Kn} \approx[v][\tau] /[L]$ where $v$ is the relevant velocity, $\tau$ is the corresponding mean-free time of flight and $L$ is the length scale of interest. For steady two-dimensional problems, we have applied the discrete ordinate method with Cartesian coordinates in the physical space and polar coordinates in the velocity space. The set of discretized governing equations are solved by using second-order upwinding finite volume method with 16th-order Gauss-Hermite half-range quadrature [28] applied to the integration over the velocity magnitude. In ES-BGK model, a Newton's algorithm [29] was applied to obtain the values of the local anisotropic Gaussian that strictly enforce mass, momentum and energy conservation at every point in the discretized spatial domain.

A grid convergence study has been carried out to determine the sensitivity to discretization of both physical and velocity spaces. For Kn approaching the free molecular limit for gas or the ballistic limit for phonons, a coarse mesh is sufficient since the intermolecular or interphonon collisions are less important compared to boundary scattering. For Kn close to the subcontinuum limit, a finer mesh is required to capture the gradients due to collisional relaxation, and to resolve sharp near-wall gradients.

\section{MODEL VERIFICATION}

\section{A. 1D gas-phase heat transfer}

The first verification case is the one-dimensional gasphase heat transfer problem. Results based on the present Boltzmann solver have been compared to experimental data for Helium in reference [30]. In the experiment, Helium gas fills the gap between two plates with constant wall temperatures of 79 and $294 \mathrm{~K}$. The thermal accommodation coefficients for both walls were measured to be 0.58 . In numerical modeling, the same conditions were used and two cases with nominal Knudsen numbers of 0.075 and 0.399 have been computed. Comparisons are plotted in Fig. 3. It can be seen that numerical results agree well with experimental data not only for the slip case with $\mathrm{Kn}<0.1$, but also for the transitional case with a larger Knudsen number. In general, the Boltzmann equation is applicable to the whole range of Knudsen numbers from continuum to free molecular.

\section{B. 1D Gas-Phonon Heat Transfer}

In order to examine the presented gas-phonon coupling model, we have considered a one-dimensional heat transfer case with gas-solid interface as shown in Fig. 2. The solution of the phonon BTE in solids has been the subject of many publications. In particular, the reader is referred to Refs. $[21,31]$ for a detailed description of the various existing phonon transport models and their transient and steady state solutions. The results of [19] discuss in detail this particular case in terms of the interfacial thermal resistance, temperature drop in the solid and gas phase and verification against asymptotic analytical solutions. Here, we detail a few results for completeness. The lengths of simulation domains for the gas and solid phase are equal to $L\left(=L_{r e f}\right)$ as shown in Fig. 2, 


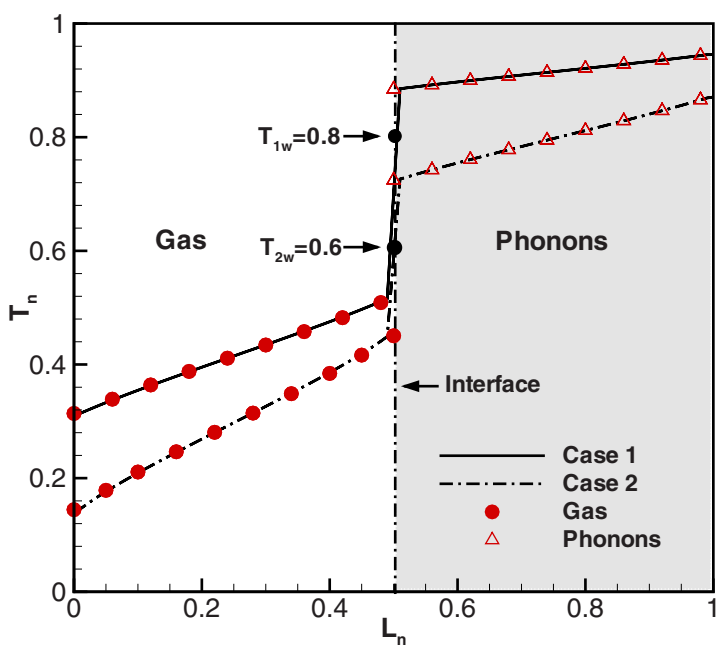

FIG. 4. (Color online) 1D gas-phonon heat transfer. Case 1: $\mathrm{Kn}_{g}=1.0, \mathrm{Kn}_{p}=1.0 ;$ Case 2: $\mathrm{Kn}_{g}=0.2, \mathrm{Kn}_{p}=1.0 . T_{n}=\left(T-T_{L}\right) /\left(T_{H}\right.$ $\left.-T_{L}\right)$.

and the temperature boundary condition is applied at the outer gas and phonon main boundaries while the interfacial temperature is directly computed from Eqs. (25)-(29). The value of $T_{L}$ is fixed at $300 \mathrm{~K}$ and heat conduction is simulated for a very small temperature difference $T_{H}-T_{L} \ll T_{L}$.

Considering $\mathrm{Kn}_{p}$ fixed at 1.0, two cases with $\mathrm{Kn}_{g}$ equals to 1.0 and 0.2 are compared in Fig. 4. For case 1, the normalized interface temperature, $T_{1 w}$, is approximately 0.8 . As $\mathrm{Kn}_{g}$ decreases, the gas phase temperature jump in case 2 decreases and the normalized temperature, $T_{2 w}$, reduced to about 0.6 .

\section{THERMAL TRANSPIRATION}

For a closed thermal transpiration system, initially, the pressure is constant everywhere and the gas flow is driven by the temperature gradient moving from cold to hot end, as shown in Fig. 5(a). The subscript 1 and 2 refer to the conditions at the entrance and the exit of the channel, while $I$ and II refer to the conditions at chamber inlet and outlet. In the process of gas molecules being redistributed, there will be a

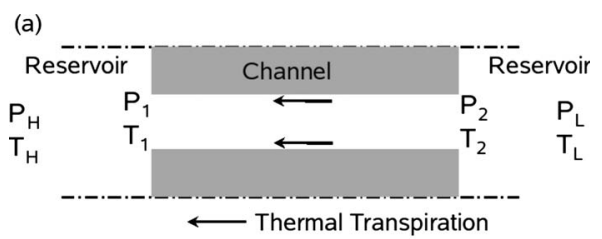

(b)

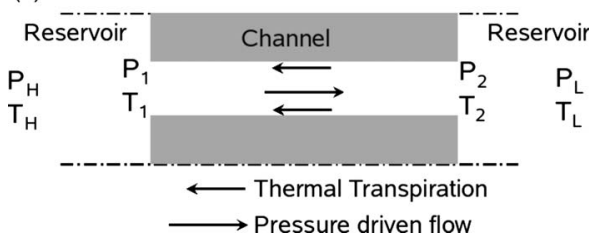

FIG. 5. Evolution of thermal transpiration flows in a closed system. (a) Initial stage: $P_{I}=P_{I I}$, thermal transpiration flow dominates; (b) Final stage: $P_{I}>P_{I I}$, the net flow approaches to zero. pressure gradient built up gradually in the system. As a consequence, in addition to thermal creep flow from the cold to the hot end, there will be an induced Poiseuille flow moving in the reverse direction as shown in Fig. 5(b). Eventually the system achieves a steady state and the net flow becomes zero.

In the presence of a small temperature and pressure gradient, the reduced mass flow rate $Q_{m}$ can be written as [32],

$$
Q_{m}=-Q_{p} v+Q_{T} \tau,
$$

where $v=L_{r e f} d P / P d x$ and $\tau=L_{r e f} d T / T d x$ are the imposed dimensionless pressure and temperature gradient in the longitudinal direction respectively. Flow coefficients $Q_{T}$ and $Q_{P}$ are functions of the local Knudsen number in the gas phase. For zero net flow, by substituting $v$ and $\tau$ into Eq. (30) and integrating over the channel length, one can get,

$$
\frac{P_{1}}{P_{2}}=\left(\frac{T_{1}}{T_{2}}\right)^{\gamma},
$$

where $\gamma=Q_{T} / Q_{P}$ represents the system compression efficiency that depends on local Knudsen numbers, channel length-to-height ratio, $L / H$, molecular mass of the gas, $m_{g}$, thermal conductivity, $k_{g}$, viscosity, $\mu_{g}$, and gas-solid interaction parameters. In some references, $\gamma$ is also referred as the thermomolecular pressure difference (TMPD) [17].

Considering free-molecular cases, at steady state, the molecular fluxes $\dot{m}$ entering and exiting the connecting channels from each reservoir must be balanced,

$$
\begin{gathered}
\dot{m}_{\text {hot }}=\dot{m}_{\text {cold }} \\
\dot{m}_{i}=\frac{n_{i} v_{i, \text { ave }}}{4}, \quad i=\text { hot }, \text { cold },
\end{gathered}
$$

where $v_{\text {ave }}$ is the average molecular velocity. According to ideal gas law and the kinetic theory,

$$
n_{i}=\frac{p_{i}}{R T_{i}}, \quad v_{i, a v e}=\frac{8 R T_{i}}{\pi}
$$

Substituting Eq. (32)-(34) into Eq. (31), we can get the following relation of pressure to temperature under asymptotic free-molecular conditions,

$$
\frac{P_{1}}{P_{2}}=\left(\frac{T_{1}}{T_{2}}\right)_{F M}^{\gamma}, \quad \gamma_{F M}=0.5
$$

However, for continuum cases, we know that zero net flow implies a zero pressure gradient as long as buoyancy induced effects are negligible, no matter whether there is temperature gradient or not. Therefore,

$$
\frac{P_{1}}{P_{2}}=\left(\frac{T_{1}}{T_{2}}\right)_{\text {cont }}^{\gamma}, \quad \gamma_{\text {cont }}=0 .
$$

In the present work, we are interested in gas flows that fall in the transitional regime. Specific gas properties and simulation conditions involved in simulations have been summarized in Table I. The schematic of the geometry is shown in Fig. 6. 
TABLE I. Summary of flow conditions.

\begin{tabular}{|c|c|}
\hline Parameter & Type/Range \\
\hline Gas (perfect gas, power-law) & $\mathrm{N}_{2}, \mathrm{H}_{2}$ \\
\hline Channel length-to-height ratio, $L_{c h} / H_{c h}$ & 5,10 \\
\hline Normalized hydraulic diameter, $L_{r e f} / H_{c h}$ & $2($ for $2 \mathrm{D})$ \\
\hline Reservoir length-to-height ratio, $L / H$ & $0.5,1,2$ \\
\hline Membrane thickness to channel height, $H_{m} / H_{c h}$ & $1.5,4,9$ \\
\hline Temperature ratio, $T_{H} / T_{L}\left(T_{L}=300 \mathrm{~K}\right)$ & $2,1.5,1.1$ \\
\hline Outlet pressure ratio (chamber), $P_{H} / P_{L}$ & $1.03-1.36$ \\
\hline Outlet pressure ratio (channel), $P_{2} / P_{1}$ & $1.03-1.25$ \\
\hline Thermal conductivity ratio, $k_{g, T=300 K} / k_{p}$ & $0.0018-3.6$ \\
\hline Gas phase $\mathrm{Kn}_{g}$ & $0.5,2.5,5.0,25$ \\
\hline Solid phase $\mathrm{Kn}_{p}$ & $0,1.5,15$ \\
\hline
\end{tabular}

\section{RESULTS AND DISCUSSION}

\section{A. Gas-phonon coupling effects}

Obtaining precise temperature distributions along channels is a key issue in the analysis of thermal transpiration problems. However, technical difficulties have set great limitations on experimental approaches, and it is rarely possible to have high-resolution temperature measurements at the submicron sized channel surfaces. Therefore, assumptions of boundary conditions need to be made for both analytical models and numerical simulations, when considering the gas phase only. In addition, the actual temperature jumps at interfaces consist of contributions from both gas and phonon subcontinuum effects, i.e., $\Delta T_{w}=\Delta T_{w, g}+\Delta T_{w, p}$.

In present work, the coupled modeling is applied to a closed thermal transpiration system filled with a perfect gas with $\operatorname{Pr}=2 / 3$, as shown in Fig. 6. Since solid membranes are also included in the computational domain, boundary conditions now become explicit for the entire system, i.e., either symmetry or known constant temperature of reservoirs. The unknown gas-solid interfaces, which correspond to Wall $_{1-3}$ in the plot, can be solved through coupling directly. For convenience, we denote the length and height of reservoirs as $L$ and $H$, the length and height of channels as $L_{c h}$ and $H_{c h}$, and the membrane thickness as $H_{m}=H-H_{c h}$.

Further manipulations on gas-solid interfaces lead to three particular models: uncoupled, weakly coupled and strongly coupled. In Table II, specific interface conditions for each model have been summarized. It is easy to see that the un-

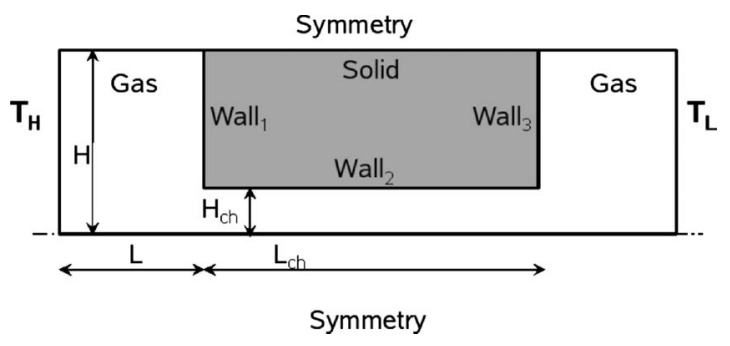

FIG. 6. Schematic of thermal transpiration in a closed system. $L_{c h} / H_{c h}=5, L / L_{c h}=0.5, H / H_{c h}=4.0$.
TABLE II. Summary of gas-phonon coupling interface conditions.

\begin{tabular}{lccc}
\hline \hline Models & $\mathrm{Wall}_{1}$ & $\mathrm{Wall}_{3}$ & $\mathrm{Wall}_{2}$ \\
\hline Uncoupled & $T_{H}$ & $T_{L}$ & Linear \\
Weakly coupled & $T_{H}$ & $T_{L}$ & Coupled \\
Strongly coupled & Coupled & Coupled & Coupled \\
\hline \hline
\end{tabular}

coupled case will give the same results as those of previous studies, which have considered the gas phase only. In contrast, the strongly coupled case simply implies that all three interfaces will be solved through coupling.

In the weakly coupled model, the temperature distribution of $\mathrm{Wall}_{2}$ will be obtained from simulations, while those of Wall $_{1}$ and $\mathrm{Wall}_{3}$ are maintained at fixed values. This case represents the scenario of enforcing extra heat sources or sinks at side walls of reservoirs more closely.

In Figs. 7 and 8, the uncoupled and the weakly coupled cases have been compared first. The relative temperature gradient $\Delta T \times L_{r e f} /\left(T_{L} \times L_{c h}\right)$ is 0.4 , the solid-to-gas thermal conductivity ratio $k^{*}\left(=k_{g} / k_{p}\right)$ is 0.18 , and the gas-phase Knudsen number $\mathrm{Kn}_{g}$ is 2.5. It implies that: a) linear approximation of temperature gradient is no longer valid, and b) the rarefaction effects are prominent.

For gas, thermal conductivities at standard conditions (1 atm, $298.15 \mathrm{~K}$ ) are about $0.024 \mathrm{~W} / \mathrm{mK}$ for air (nitrogen, oxygen), $0.016 \mathrm{~W} / \mathrm{mK}$ for argon, $0.142 \mathrm{~W} / \mathrm{mK}$ for helium, 0.168 $\mathrm{W} / \mathrm{mK}$ for hydrogen and $0.016 \mathrm{~W} / \mathrm{mK}$ for water vapor at (398.15 K). For solid, the typical semiconductor materials cannot be put to use in practical thermal transpiration applications due to their high thermal conductivity. For example, thermal conductivity is around $149 \mathrm{~W} / \mathrm{mK}$ for bulk crystalline silicon. Instead, low-thermal conductivity materials are usually employed to maintain temperature gradients that can drive the fluid flow. Recent reports include silica aerogel, whose thermal conductivity is as low as $0.02 \mathrm{~W} / \mathrm{mK}$, $\mathrm{W} / \mathrm{Al}_{2} \mathrm{O}_{3}$, whose thermal conductivity is $0.6 \mathrm{~W} / \mathrm{mK}[33]$, $\mathrm{WSe}_{2}$, whose thermal conductivity is $0.05 \mathrm{~W} / \mathrm{mK}$ [34], and zeolite, whose thermal conductivity is about $0.5 \mathrm{~W} / \mathrm{mK}$ [18]. Another material that holds promise for thermal transpiration devices is rough silicon nanowire arrays, which has demonstrated thermal conductivities below $1 \mathrm{~W} / \mathrm{mK}$ at room temperatures and enhanced thermoelectric performance [35].

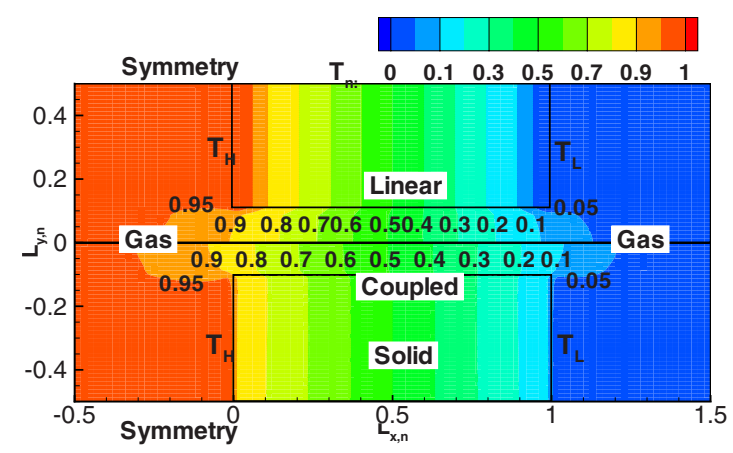

FIG. 7. (Color online) Temperature maps for uncoupled and coupled cases. $T_{n}=\left(T-T_{L}\right) /\left(T_{H}-T_{L}\right)$. 


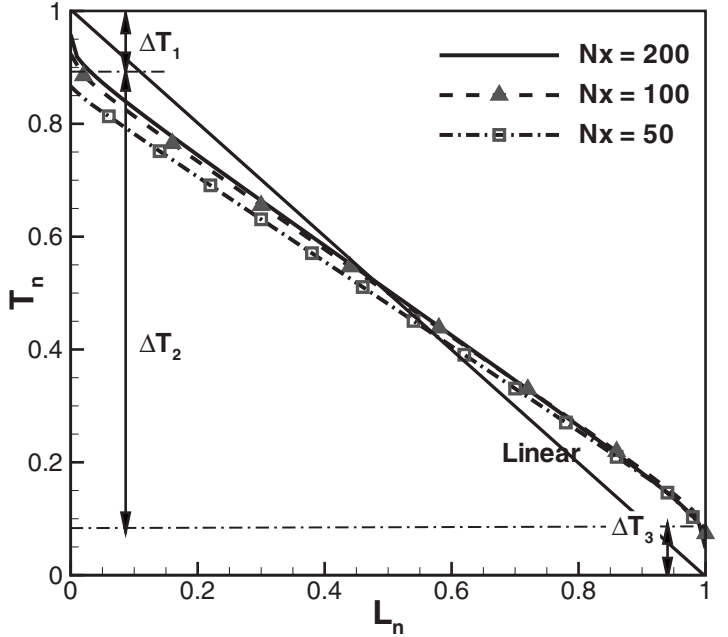

FIG. 8. Wall temperature distributions for grids. $T_{n}=(T$ $\left.-T_{L}\right) /\left(T_{H}-T_{L}\right)$.

The thermal conductivity ratio we chose for the simulations is therefore a reasonably medium value.

In the coupled model, size effects for solid membranes also need to be quantified in spite of being more subtle than gas. According to Refs. [16,17], typical values of the thermal conductivity and structural parameters of a typical aerogel are used to calculate the nominal Knudsen number for membranes $\mathrm{Kn}_{p}$. Based on Eqs. (15) and (16) this yields $\mathrm{Kn}_{p}$ $=1.5$. This says that the associated temperature jumps at the boundaries are contributed by both phonons and gas molecules thereby increasing the estimate of thermal contact resistance that would be predicted if temperature drop is limited to the gas phase only.

As shown in Fig. 7, the temperature field emerging out of a coupled simulation is significantly different from when a linear temperature gradient is assumed. As compared to the uncoupled situation, contour lines are denser near Wall $_{1,3}$ and sparser along $\mathrm{Wall}_{2}$ in the coupled case. As shown in Fig. 8, the actual temperature differences $\Delta T_{2}$ of thermal transpiration in coupled cases are less than the difference

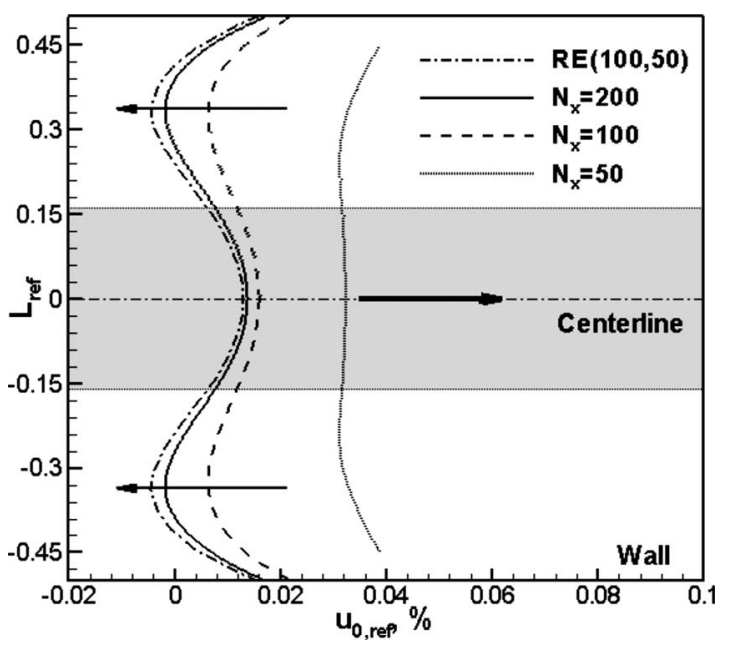

FIG. 9. Velocity profiles for different grids and Richardson extrapolation. $u_{0, r e f}, \%=100 \times u / \sqrt{2 R T}$.

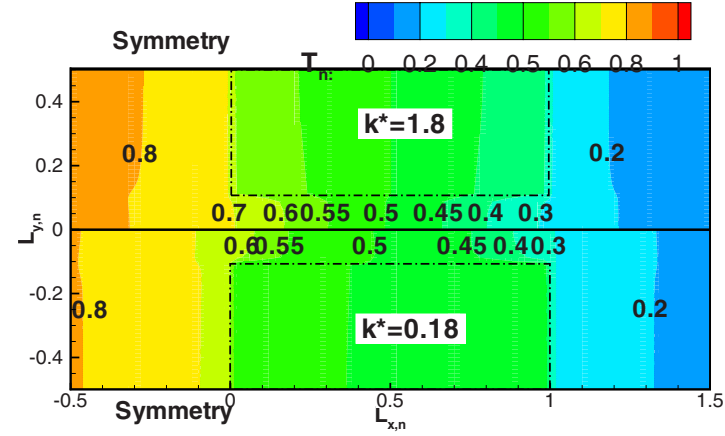

FIG. 10. (Color online) Temperature maps for strongly coupled cases. Top: $k^{*}=1.8$. Bottom: $k^{*}=0.18 T_{n}=\left(T-T_{L}\right) /\left(T_{H}-T_{L}\right)$.

between reservoirs. Hence, one may not disregard the fact that a continuum description of thermal transport is not valid in the solid and that thermal contact resistance needs to be considered.

At equilibrium, it is known that the system becomes steady, as the pressure-driven Poiseuille flow and thermal creep flow [36] are balanced. However, in consequence of flow superposition, there will be a vortical flow inside the whole system, although at a very low speed.

As shown in Fig. 9, channel flows tend to move from left to right near walls and reverse the direction near centerlines. The main reason is that velocity profiles of Poiseuille flows inside channels are parabolic. Therefore, pressure-driven flow has a stronger impact over thermal transpiration near centerlines. We use the low-speed flow example to examine the grid sensitivity of the computational results. Compared to temperature and pressure profiles, the velocity is very sensitive to the change of grid sizes, as seen in Figs. 8 and 9.

The importance of gas-solid interaction has already been demonstrated in the above analysis. However, there is another unsolved question about coupling, which is the necessity of having thermal sources and sinks enforced at interfaces Wall ${ }_{1}$ and $\mathrm{Wall}_{3}$. In other words, what would be the change of the compression efficiency for a closed thermal creep system if we consider the strongly coupled model instead? To evaluate the role of the additional heat exchange, we have simulated cases using the strongly coupled model at different thermal conductivity ratios. As shown in Fig. 10, the actual temperature difference responsible in driving thermal transpiration is smaller than the weakly coupled case shown earlier for $k^{*}=0.18$, as the solid membrane becomes the major heat transfer pathway compared to the gas. For the case with $k^{*}=1.8$, temperature gradient increases and heat flux through membranes reduces. Therefore, ideal solid membranes really need to have extremely low thermal conductivity.

TABLE III. Summary of coupling effects.

\begin{tabular}{lccc}
\hline \hline Variations & $T_{2} / T_{1}$ & $P_{1} / P_{2}$ & $\gamma$ \\
\hline Uncoupled, $k^{*}=0.18$ & 1.749 & 1.252 & 0.401 \\
Weakly coupled, $k^{*}=0.18$ & 1.646 & 1.218 & 0.396 \\
Strongly coupled, $k^{*}=0.18$ & 1.269 & 1.093 & 0.375 \\
Strongly coupled, $k^{*}=1.8$ & 1.348 & 1.122 & 0.385 \\
\hline
\end{tabular}




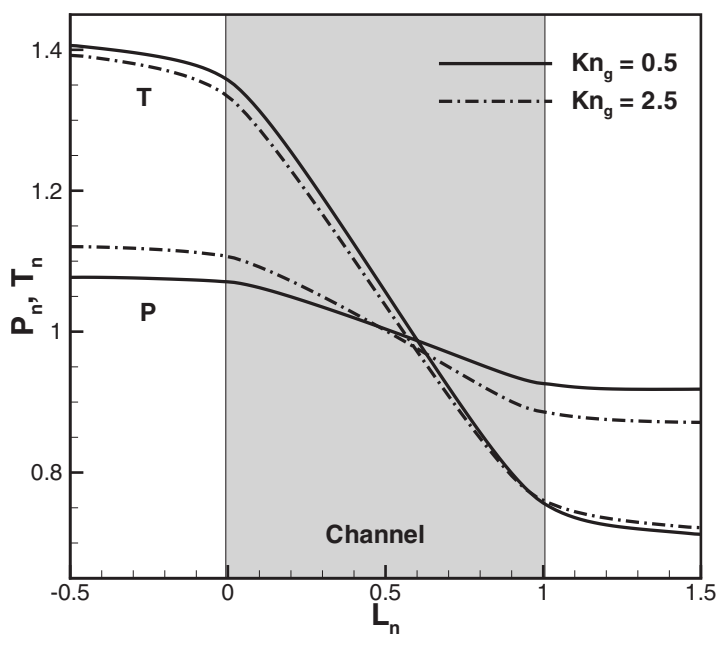

FIG. 11. Centerline normalized temperature and pressure distributions at different $\mathrm{Kn}_{g} . \mathrm{Kn}_{p}=1.5, T_{n}=T / \sqrt{T_{H} T_{L}}, P_{n}=P / P_{L_{n}}=0.5$.

A summary of calculated compression coefficients for cases with different interfacial conditions is given in Table III. The efficiency of the weakly and strongly coupled cases is lower than that of the uncoupled simulations (but the difference is small), which suggests that the gas molecules are imposed to an effective thermal gradient which is smaller than one that assumes a simple linear gradient. For convenience, we will refer to "weakly coupled" cases by simply using terminology of "coupled" in the following discussions.

\section{B. Knudsen number effects}

According to the theoretical analysis, the compression coefficient, $\gamma$, varies from zero in the continuum limit to 0.5 in the free-molecular limit. The pressure and temperature profiles corresponding to $\mathrm{Kn}_{g}$ of 0.5 and 2.5 are shown in Fig. 11. In these simulations $\mathrm{Kn}_{p}$ is kept the same. These results clearly indicate the trend in the compression coefficient. At $\mathrm{Kn}_{g}=0.5, P_{1} / P_{2}$ is quite small compared to the case of $\mathrm{Kn}_{g}=2.5$ for almost the same values of $T_{1} / T_{2}$. As stated ear-

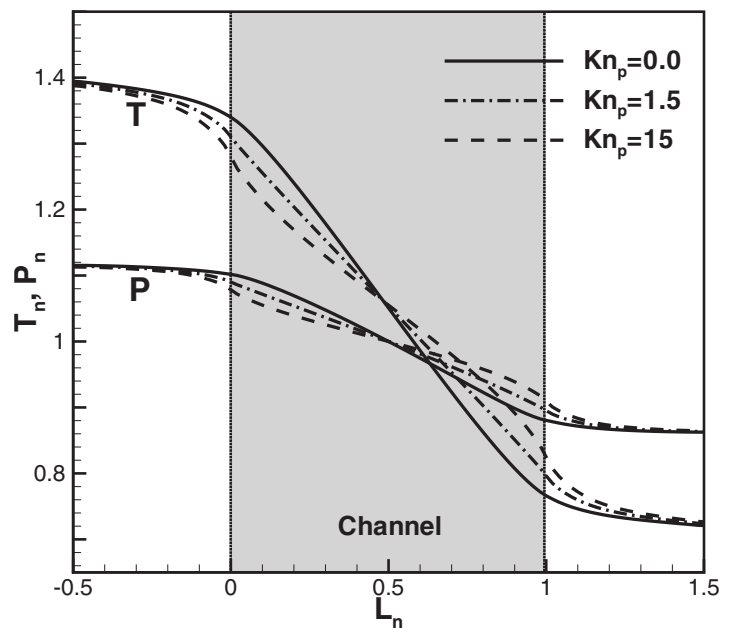

FIG. 12. Centerline temperature and pressure distributions at different $\mathrm{Kn}_{p} . \mathrm{Kn}_{g}=2.5, T_{n}=T / \sqrt{T_{H} T_{L}}, P_{n}=P / P_{L_{n}=0.5}$.
TABLE IV. Summary of Knudsen number effects.

\begin{tabular}{llll}
\hline \hline Knudsen Number & $T_{2} / T_{1}$ & $P_{1} / P_{2}$ & $\gamma$ \\
\hline $\mathrm{Kn}_{g}=0.5, \mathrm{Kn}_{p}=1.5$ & 1.795 & 1.146 & 0.233 \\
$\mathrm{Kn}_{g}=2.5, \mathrm{Kn}_{p}=1.5$ & 1.753 & 1.249 & 0.396 \\
$\mathrm{Kn}_{g}=25, \mathrm{Kn}_{p}=1.5$ & 1.629 & 1.267 & 0.485 \\
$\mathrm{Kn}_{p}=0.0, \mathrm{Kn}_{g}=2.5$ & 1.749 & 1.252 & 0.401 \\
$\mathrm{Kn}_{p}=1.5, \mathrm{Kn}_{g}=2.5$ & 1.646 & 1.218 & 0.396 \\
$\mathrm{Kn}_{p}=15, \mathrm{Kn}_{g}=2.5$ & 1.552 & 1.186 & 0.388 \\
\hline \hline
\end{tabular}

lier, the integrated pumping efficiency requires not only high compression ratios but also needs to maximize the mass-flow rate. In addition, a hint from the coupling implies that as membrane sizes are made smaller and $\mathrm{Kn}_{g}$ increases, significant rarefaction may be seen in thermal transport in the solid membrane as well. The actual temperature gradient decreases due to significant temperature jumps at gas-solid interfaces at high $\mathrm{Kn}_{p}$, as shown in Fig. 12. Quantitative effects on $\gamma$ for various values of $\mathrm{Kn}_{g}$ and $\mathrm{Kn}_{p}$ can be found in Table IV.

Based on the analysis in this section, some general criteria may be concluded for the selection of membrane material and the working gas. First, the solid phase must be able to maintain temperature differences, i.e., it must have a low thermal conductivity. Meanwhile, the solid phase Knudsen number $\mathrm{Kn}_{p}$ must be small compared to the gas-phase Knudsen number $\mathrm{Kn}_{g}$ so that the inefficiency due to large interfacial temperature jump in the solid can be relieved. In addition, under the same thermal and pressure conditions, the particular gas will have a higher $\mathrm{Kn}_{g}$ for a larger viscosity coefficient. In general, gas viscosity is temperature dependent. According to the power-law model given in Eq. (5), the viscosity increases as temperature increases. Therefore, the gas pumping system based on thermal transpiration mechanism should have better performances in low temperature environments.

\section{Interface conditions}

The gas-phase thermal accommodation coefficient $\sigma_{T, g}$ and phonon transimissivity $\alpha_{p}$ have been assumed unity in all previous cases. Thus, they correspond to situations with maximum heat flux through interfaces. In this section, we

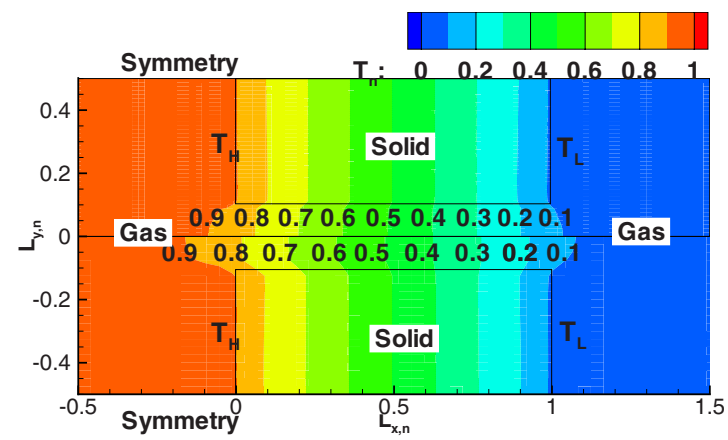

FIG. 13. (Color online) Temperature contours at different $\sigma_{T}$ (assuming $\left.\sigma_{T, g}=\alpha_{p}\right)$. $\mathrm{Kn}_{g}=2.5, \mathrm{Kn}_{p}=1.5, T_{n}=\left(T-T_{L}\right) /\left(T_{H}-T_{L}\right)$. 


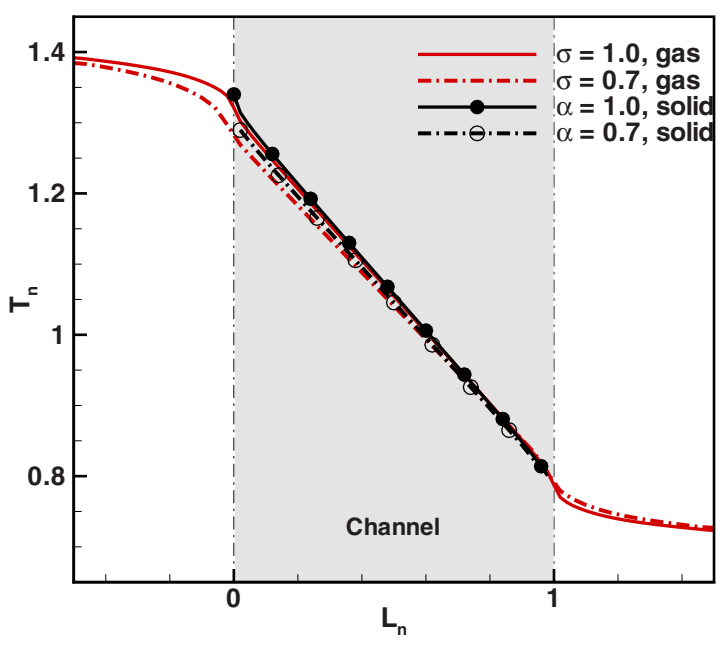

FIG. 14. (Color online) Interface temperature and pressure distributions at different $\sigma_{T}$ (assuming $\sigma_{T, g}=\alpha_{p}$ ). $\mathrm{Kn}_{g}=2.5, \mathrm{Kn}_{p}=1.5$, $T_{n}=T / \sqrt{T_{H} T_{L}}, P_{n}=P / P_{L_{n}=0.5}$.

will present a nonperfect case with $\sigma_{T, g}=\alpha_{p}=0.7$. As shown in Figs. 13 and 14, the actual temperature difference responsible in driving thermal transpiration along the channel decreases due to smaller heat flux through gas-solid interfaces. Surface condition effects on the compression coefficient $\gamma$ can be found in Table $\mathrm{V}$ and are seen to be strongly suppressed when the accommodation coefficient less than unity.

\section{Comparisons with analytical models}

In this section, numerical results based on coupled modeling will be compared with previous analytical solutions. First, there is a linear analytical model developed by Loyalka et al. in 1975 [37], which is based on approximations of the BGK model and Maxwellian boundary conditions of diffusespecular reflection with an assumption of linear temperature and pressure gradient. Comparisons between simulations and analytical results have been shown in Table VI. In the presence of large temperature difference, the linear assumption gradient approximation gives a better result for $\mathrm{Kn}_{g}=2.5$, where the actual $\Delta T$ is smaller, than for $\mathrm{Kn}_{g}=0.5$ as expected.

A more sophisticated analytical model suggested by Sharipov et al. [32] has made some progress on the nonlinear effects in thermal transpiration. The analysis considers thermal transpiration at an arbitrary temperature ratio in a tube based on the S-model [38] for the diffuse specular gassurface interaction in the range of the rarefaction parameter $\delta$ from 0.005 to 50 . The rarefaction parameter is inversely proportional to the Knudsen number,

TABLE V. Summary of surface condition effects.

\begin{tabular}{lccc}
\hline \hline Accommodation coefficient & $T_{2} / T_{1}$ & $P_{1} / P_{2}$ & $\gamma$ \\
\hline$\sigma_{T, g}=\alpha_{p}=1.0$ & 1.646 & 1.218 & 0.396 \\
$\sigma_{T, g}=\alpha_{p}=0.7$ & 1.600 & 1.190 & 0.370 \\
\hline \hline
\end{tabular}

TABLE VI. Comparison with analytical solutions [37], $\Delta$ $=\left|\gamma_{\text {ref }} / \gamma_{\text {coupled }}-1\right|$

\begin{tabular}{lcccc}
\hline \hline Case & $\mathrm{Kn}_{g}$ & Ref. [37] & Coupled & $\Delta, \%$ \\
\hline$\Delta T / T_{L}=0.4 L_{c h} / 2 H_{c h}$ & & & & \\
$\sigma_{T, g}=1.0$ & 2.5 & 0.37 & 0.396 & 6.5 \\
$\sigma_{T, g}=1.0$ & 0.5 & 0.19 & 0.233 & 18 \\
\hline \hline
\end{tabular}

$$
\delta=\frac{p L_{c h}}{\mu} \sqrt{\frac{1}{2 R T}}=\frac{\sqrt{\pi}}{2} \frac{1}{\mathrm{Kn}_{g}} .
$$

Assuming $\Delta P / P_{1} \ll L_{c h} / H_{c h}$ and $\Delta T / T_{1} \ll L_{c h} / H_{c h}$, it gives,

$$
\delta_{\text {ave }}=\delta_{1} \frac{1+\left(T_{2} / T_{1}\right)^{\gamma}}{1+T_{2} / T_{1}} .
$$

Comparisons between the coupled simulations and the nonlinear analytical model have been summarized in Table VII. For perfect surface conditions, the compression efficiencies agreed well over a range of rarefaction parameters with differences less than $4 \%$. However, for the case with $\sigma_{T, g}$ $=0.7$, the discrepancy rises to about $11 \%$. One reason for the discrepancy is because the analytical model doesn't consider the contribution of temperature jumps at walls due to the non-unity phonon transimissivity $\alpha_{p}$ in the membrane.

\section{CONCLUSIONS}

Numerical modeling of the coupled gas-phonon thermal transport has been developed and applied to study thermal transpiration in a channel. Validation has been carried out by comparing simulation results with experimental data and analytical solutions. The coupled gas-phonon modeling shows that the temperature difference along the transpiration channel is diminished as compared to the overall temperature drop between the inlet and outlet reservoirs. This results in a lower compression efficiency for a thermal-transpiration

TABLE VII. Comparison with analytical solutions [32], $\Delta$ $=\left|\gamma_{\text {ref }} / \gamma_{\text {coupled }}-1\right|$

\begin{tabular}{lcccc}
\hline \hline Case & $\mathrm{Kn}_{g}$ & Ref. [32] & Coupled & $\Delta, \%$ \\
\hline$\Delta T / T_{1}=0.4 L_{c h} / 2 H_{c h}$ & & & & \\
$\sigma_{T, g}=1.0$ & 2.5 & 0.39 & 0.396 & 1.5 \\
$\sigma_{T, g}=0.7, \quad \alpha_{p}=0.7$ & 2.5 & 0.33 & 0.370 & 11 \\
$\sigma_{T, g}=1.0$ & 0.5 & 0.24 & 0.233 & 3.0 \\
$\Delta T / T_{1}=0.2 L_{c h} / 2 H_{c h}$ & & & & \\
$\sigma_{T, g}=1.0$ & 2.4 & 0.37 & 0.382 & 3.1 \\
$\Delta T / T_{1}=0.04 L_{c h} / 2 H_{c h}$ & & & & \\
$\sigma_{T, g}=1.0$ & 2.1 & 0.36 & 0.367 & 1.9 \\
\hline \hline
\end{tabular}


based Knudsen pump. A larger Knudsen number in the gasphase improves the compression efficiency. However, there is a tradeoff between the maximum pressure ratio and the maximum mass-flow-rate in practical thermal transpiration devices. Similarly, the solid-phase material and thermal properties need to be chosen to maintain continuum conditions to minimize temperature jumps at the gas-solid boundaries. Good agreements have been found by comparing results from simulations and analytical models under conditions of low temperature gradients. The coupled gasphonon thermal modeling can be exploited in analyzing the thermal transpiration open systems as well as other microscale thermally-driven gas flow phenomena and devices.

\section{ACKNOWLEDGMENTS}

This work has been supported by Computing Research Institute (CRI) at Purdue University and Department of Energy [National Nuclear Security Administration] under Award No. DE-FC52-08NA28617. The computations reported in this paper were performed on a 16-CPU SunFire 4600, awarded through Sun Microsystems, Inc. Academic Excellence under Grant No. EDUD-7824-070336-U.S.
[1] P. R. Lewis, P. Manginell, D. R. Adkins, R J. Kottenstette, D. R. Wheeler, S. S. Sokolowski, D. E. Trudell, J. E. Byrnes, M. Okandan, J. M. Bauer, R. G. Manley, and C. Frye-Mason, IEEE Sens. J. 6, 784 (2006).

[2] M. Bao and H. Yang, Sens. Actuators, A 136, 3 (2007).

[3] N. Bao, J. Wang, and C. Lu, Anal. Bioanal. Chem. 391, 933 (2008).

[4] J. Khandurina and A. Guttman, Curr. Opin. Chem. Biol. 7, 595 (2003).

[5] A. A. Alexeenko, D. A. Fedosov, S. F. Gimelshein, D. A. Levin, and R. J. Collins, Microelectromechanical Systems 15, 181 (2006).

[6] G. Pham-Van-Diep, P. Keeley, E. P. Muntz, and D. P. Weaver, in Rarefied Gas Dynamics, edited by J. Harvey and G. Lord (Oxford University Press, Oxford, 1995), Vol. 1, pp. 715-721.

[7] C. C. Wong, M. L. Hudson, D. L. Potter, and T. J. Bartel, Gas Transport by Thermal Transpiration in Micro-channels-A Numerical Study, Proceedings of ASME MEMS Conference 1998 (unpublished), Vol. DSC-66, pp. 223-228.

[8] S. E. Vargo, E. P. Muntz, G. R. Shiflett, and W. C. Tang, J. Vac. Sci. Technol. A 17, 2308 (1999).

[9] O. Reynolds, Philos (Tran. Royal Society, London, 1879).

[10] M. Knudsen, Ann. Phys. 16, 338 (1910).

[11] G. D. West, Proc. Phys. Soc. London 33, 266 (1920).

[12] T. Edmonds and J. P. Hobson, J. Vac. Sci. Technol. 2, 182 (1965).

[13] J. Setina, Metrologia 36, 623 (1999).

[14] O. Sazhin, A. Kulev, S. Borisov, and S. Gimelshein, Vacuum 82, 20 (2007).

[15] S. Vargo and E. P. Muntz, A Simple Micromechanical Compressor and Vacuum Pump for Flow Control and Other Distributed Applications, 34th Aerospace Sciences Meeting and Exhibit 1996 (unpublished).

[16] E. P. Muntz, Y. Sone, K. Aoki, S. Vargo, and M. Young, J. Vac. Sci. Technol. A 20, 214 (2002).

[17] Y. Han, E. P. Muntz, A. A. Alexeenko, and M. Young, Nanoscale Microscale Thermophys. Eng. 17, 151 (2007).
[18] N. K. Gupta and Y. B. Gianchandani, Appl. Phys. Lett. 93, 193511 (2008).

[19] D. Singh, X. Guo, A. A. Alexeenko, J. Murthy, and T. S Fisher, J. Appl. Phys. 106, 024314 (2009).

[20] L. Mieussens and H. Struchtrup, Phys. Fluids 16, 2797 (2004).

[21] J. Y. Murthy, S. V. J. Narumanchi, J. A. Pascual-Gutierrez, T. Wang, C. Ni, and S. R. Mathur, Int. J. Multiscale Comp. Eng. 3, 5 (2005)

[22] M. N. Kogan, Rarefied Gas Dynamics (Plenum Press, New York, 1969).

[23] P. Andries, P. Le Tallec, J.-P. Perlat, and B. Perthame, Eur. J. Mech. B/Fluids 19, 813 (2000).

[24] A. A. Alexeenko, S. F. Gimelshein, E. P. Muntz, and A. D. Ketsdever, Int. J. Therm. Sci. 45, 1045 (2006).

[25] F. P. Incropera and D. P. DeWitt, Fundamentals of Heat and Mass Transfer (John Wiley \& Sons Inc., New York, 2006).

[26] G. A. Bird, Molecular Gas Dynamics and the Direct Simulation of Gas Flows (Oxford University Press, New York, 1994).

[27] G. Chen, Phys. Rev. B 57, 14958 (1998).

[28] B. Shizgal, J. Comput. Phys. 41, 309 (1981).

[29] W. H. Press, S. A. Teukolsky, W. T. Vetterling, and B. P. Flannery, Numerical Recipes in Fortran 90 (Cambridge University Press, Cambridge, Engalnd, 2001).

[30] D. C. Wadsworth, Phys. Fluids A 5, 1831 (1993).

[31] A. A. Joshi and A. Majumdar, J. Appl. Phys. 74, 31 (1993).

[32] F. M. Sharipov, J. Vac. Sci. Technol. A 14, 2627 (1996).

[33] R. M. Costescu, D. G. Cahill, F. H. Fabreguette, Z. A. Sechrist, and S. M. George, Science 303, 989 (2004).

[34] C. Chiritescu, D. G. Cahill, N. Nguyen, D. Johnson, A. Bodapati, P. Keblinski, and P. Zschack, Science 315, 351 (2007).

[35] A. I. Hochbaum, R. Chen, R. D. Delgado, W. Lian, E. C. Garnett, M. Najarian, A. Majumdar, and P. Yang, Nature (London) 451, 163 (2008).

[36] V. Sofonea, Phys. Rev. E 74, 056705 (2006).

[37] S. K. Loyalka, J. Chem. Phys. 63, 4054 (1975).

[38] E. M. Shakhov, Fluid Dyn. 3, 112 (1968). 